\title{
MODELOS ESTATÍSTICOS INDICADORES DE COMPORTAMENTOS ASSOCIADOS A BEM-ESTAR TÉRMICO PARA MATRIZES PESADAS
}

\author{
DOUGLAS D. SALGADO ${ }^{1}$, IRENILZA DE A. NÄ̈̈ ${ }^{2}$, DANILO F. PEREIRA ${ }^{3}$, \\ DANIELLA J. DE MOURA ${ }^{4}$
}

\begin{abstract}
RESUMO: O estudo de reprodutoras de frangos de corte é de grande importância para o País, uma vez que o frango de corte é um dos maiores itens agropecuários em exportação, sendo o Brasil o segundo maior exportador mundial dessa carne. O comportamento animal é conceituado como reflexo do efeito da interação de diversos fatores, entre os quais o ambiental. Dessa forma, o ambiente interno do galpão de produção constitui um dos elementos que fornecem indicações sobre o conforto térmico das aves. O comportamento de matrizes expressa, sob a forma de padrões específicos, a saúde e o bem-estar dessas aves. Este trabalho teve como objetivo a aplicação dos modelos estatísticos preditivos, por meio da construção de cenários, apresentando resultados do conforto animal perante diversas condições ambientais. $O$ trabalho foi desenvolvido com dados coletados em ambiente controlado, utilizando a linhagem Hybro- $\mathrm{PG}^{\circledR}$, submetida a diferentes níveis de temperatura ambiente, tipos padronizados de ração e idade. Foram procedidas as análises descritiva e exploratória e, posteriormente, a modelagem, utilizando as Equações de Estimação Generalizadas (EEG). A pesquisa permitiu o desenvolvimento de indicadores de bem-estar térmico por meio das equações dos modelos estatísticos de predição, sob os distintos cenários estudados.
\end{abstract}

PALAVRAS-CHAVE: avicultura, bem-estar, modelos estatísticos.

\section{STATISTIC MODELS OF BEHAVIOR INDICATOR ASSOCIATED WITH THERMAL WELFARE FOR FEMALE BROILER BREEDER}

\begin{abstract}
The study of female broiler breeders is of great importance for the country as poultry production is one of the largest export items, and Brazil is the second largest broiler meat exporter. Animal behavior is known as a response to the effect of several interaction factors among them the environment. In this way the internal housing environment is an element that gives hints regarding to the bird's thermal comfort. Female broiler breeder behavior, expresses in form of specific pattern the bird's health and welfare. This research had the objective of applying predictive statistical models through the use of simulation, presenting animal comfort scenarios facing distinct environmental conditions. The research was developed with data collected in a controlled environment using Hybro - $\mathrm{PG}^{\circledR}$ breeding submitted to distinct levels of temperature, three distinct types of standard ration and age. Descriptive and exploratory analysis were proceeded, and afterwards the modeling process using the Generalized Estimation Equation (GEE). The research allowed the development of the thermal comfort indicators by statistical model equations of predicting female broiler breeder behavior under distinct studied scenarios.
\end{abstract}

KEYWORDS: poultry production, welfare, statistical models.

\footnotetext{
${ }^{1}$ Estatístico, Mestre em Engenharia Agrícola, Doutorando, FEAGRI-UNICAMP, Campinas - SP.

${ }^{2}$ Eng $^{\mathrm{a}}$ Civil, Professora Colaboradora Voluntária, FEAGRI-UNICAMP, Campinas - SP.

${ }^{3}$ Eng $^{\text {o }}$ Agrícola, Professor Assistente Doutor, UNESP-Tupã - SP.

${ }^{4}$ Eng ${ }^{a}$ Agrônoma, Professora Assistente Doutora, FEAGRI-UNICAMP, Campinas - SP.

Recebido pelo Conselho Editorial em: 20-10-2006

Aprovado pelo Conselho Editorial em: 22-8-2007
} 


\section{INTRODUÇÃO}

O mercado avícola nacional vem-se modificando em função dos mercados compradores e das exigências de produção. O segmento de produção de matrizes é hoje um setor altamente especializado, principalmente em função do uso de tecnologia para a produção de pintinhos de um dia, direcionada aos setores de produção de frangos de corte e aves de postura. As matrizes pesadas fêmeas, em fase de postura, por serem maiores e com metabolismo mais intenso, são menos tolerantes ao calor do que as aves leves. A influência do ambiente físico na resposta comportamental de reprodutores tem sido descrita na literatura (KEER-KEER et al., 1996; McGARY et al., 2003; CHENG \& MUIR, 2005).

Existe grande número de variáveis influenciando o microclima dentro de uma instalação avícola, e conhecer sua interferência na produtividade é determinante para melhor compreensão dos fenômenos ambientais (NÄ̈̈S, 1994).

O conforto térmico no interior de instalações avícolas interfere consideravelmente na produção das aves. $\mathrm{O}$ excesso de frio e, principalmente, o excesso de calor, revertem em menor produtividade, afetando também seu crescimento e saúde, o que pode levar a situação extrema, como o acréscimo da mortalidade dos lotes. A faixa de termoneutralidade é aquela na qual a ave utiliza o mínimo de energia para se termorregular, variando conforme o tamanho do animal, manejo, aspectos nutricionais e a estrutura física da instalação (ESMAY, 1982; TEETER \& BELAY, 1996; PEREIRA, 2003; BROWN-BRANDL, 2005).

Dependendo da magnitude e da duração do estresse térmico sofrido pelas aves, podem ocorrer desde pequenos decréscimos no ganho de peso, até prostração e morte. Esses efeitos podem resultar de desajuste no mecanismo fisiológico de termorregulação das aves, numa tentativa de compensar os efeitos do estresse térmico, aos quais as aves estejam submetidas (MITCHELL, 1987). O comportamento das aves sofre influência do ambiente térmico externo, modificando a sua resposta quanto ao bem-estar (GRAVES, 1982; DUNCAN \& MENCH, 1993). ESTEVEZ et al. (2003) observaram os comportamentos de galinhas poedeiras, em vários tamanhos de grupo, e concluíram que a dinâmica dos comportamentos agressivos foi influenciada pelo contexto da competição pelo alimento; o tamanho do grupo, por sua vez, influenciou inversamente na média de interações agressivas, sendo observadas menos agressões em grupos maiores, mantendo-se a densidade de aves, corroborando os relatos de GRAVES (1982).

Diversos trabalhos (PEREIRA, 2005; PEREIRA et al., 2005; SALGADO, 2006) desenvolveram modelos preditivos de comportamento de matrizes pesadas frente a diversos cenários constituídos em um experimento controlado, apontando a presença e a magnitude de alguns comportamentos como indicadores de bem-estar. CURTO et al. (2007), estudando o comportamento de matrizes pesadas, encontraram a existência de um padrão de preferência térmica das aves, diferenciado frente às situações de ambiente, descrito por meio do modelo probabilístico para cálculo do valor esperado de freqüência de ocorrência dos comportamentos, em função das variáveis do ambiente de alojamento. Utilizando estimações por intervalos de confiança, PEREIRA (2003) também estabeleceu relações entre o comportamento de aves poedeiras e as temperaturas críticas.

Constata-se a importância dos modelos preditivos de comportamento por fazerem ligação entre os cenários constituídos experimentalmente e o comportamento das aves (PEREIRA, 2005; PEREIRA et al., 2005; SALGADO, 2006; CURTO et al., 2007).

Ao encontro das demandas de mercado, diversos autores têm pesquisado o bem-estar animal, utilizando tecnologias complexas para os estudos do comportamento. Tais estudos têm-se mostrado mais viáveis para inferir sobre os níveis de bem-estar térmico de aves alojadas (MARÍA et al., 2004; MARCHANT et al., 2001; PEREIRA, 2003), uma vez que variáveis fisiológicas são difíceis de serem medidas em condições de campo. 
GRAVES (1982), DUNCAN \& MENCH (1993) e CAMPOS (2000) consideram o comportamento como uma possibilidade de o animal expressar sofrimento, frustração e dor, podendo ser projetado em níveis de bem-estar. A correta interpretação dessas respostas, aliada às tecnologias de precisão, constituirá o futuro do monitoramento de condições comerciais de produção.

Com o objetivo de utilizar a freqüência de determinados comportamentos como indicadores de conformo térmico, para matrizes de frango de corte, este trabalho apresentou cenários resultantes dos modelos de predição do comportamento em função de condições ambientais impostas.

\section{MATERIAL E MÉTODOS}

O experimento foi realizado em câmara climática, na Faculdade de Engenharia Agrícola, da Universidade Estadual de Campinas, FEAGRI, Campinas - SP, situada na latitude $22^{\circ} 53$ 'S e longitude $47^{\circ} 03^{\prime} \mathrm{W}$. Foram verificadas respostas comportamentais em função do ambiente. O estudo foi realizado durante nove dias, entre dezembro de 2004 e janeiro de 2005.

As condições experimentais estabelecidas estão descritas a seguir:

Aves - matrizes pesadas: neste estudo, foram utilizadas 27 matrizes (fêmeas) de frango de corte pertencentes à linhagem Hybro-PG, divididas em três grupos, com diferentes idades, sendo: nove aves com idade de 38 semanas de vida; nove aves com idades entre 46 a 51 semanas de vida, e nove aves com idade de 58 semanas de vida. As três faixas etárias foram escolhidas de acordo com os principais pontos na curva de produção.

Arraçoamento: as aves foram alimentadas com três tipos padronizados de rações $(\mathrm{r} 1, \mathrm{r} 2 \mathrm{e}$ r3), contendo diferentes composições e quantidades de energia metabolizável, correspondendo, respectivamente, a $2.810 \mathrm{kCal} \mathrm{kg}^{-1} ; 2.800 \mathrm{kCal} \mathrm{kg}^{-1}$, e $2.789 \mathrm{kCal} \mathrm{kg}^{-1 *}$. A oferta de ração foi restrita a $150 \mathrm{~g}$ por ave por dia, posta de manhã, entre $7 \mathrm{~h}$ e $8 \mathrm{~h}$, reproduzindo-se a restrição alimentar das granjas.

Programa de luz: como as aves utilizadas neste experimento estavam em diferentes estágios de produção e, portando, submetidas a diferentes programas de luz nas granjas, adotou-se o programa de luz, dentre os grupos que proporcionavam o maior período de iluminação, ou seja, $14 \mathrm{~h}$ de luz e $10 \mathrm{~h}$ de escuro.

Logística: para viabilizar a logística do experimento de entrada de grupos de matrizes pesadas regularmente na câmara climática, todas as 27 aves que foram doadas pela granja foram, primeiramente, alojadas no campo experimental do Laboratório de Ambiência I (FEAGRI), em galpões construídos em escala reduzida, durante um dia, para minimizar o estresse do transporte. Esses modelos, posteriormente, foram utilizados como salas de espera, e as matrizes receberam o mesmo tipo de manejo e alimentação das granjas.

O experimento foi realizado ao longo de nove dias e, a cada três dias, nove aves (três aves por idade) eram alocadas dentro da câmara climática dividida em três boxes, cada qual alocando um grupo de três matrizes de mesma idade e contendo um tipo de ração diferente.

Ambiente: cada grupo de matrizes pesadas foi submetido a um ambiente constante durante três dias consecutivos. Neste experimento, foram simulados três ambientes térmicos diferentes (de 13; 21; ou $35^{\circ} \mathrm{C}$ ), baseando-se em BOUSHY \& RATERINK (1985), e fixando-se a umidade relativa em $75 \%$, baseando-se em TINÔCO (2001). O ambiente desejado era programado no painel de controle eletromecânico da câmara climática.

Monitoramento remoto: as aves tiveram suas expressões comportamentais filmadas por três câmeras de vigilância de ângulo aberto, colocadas no teto da câmara climática, uma sobre cada

\footnotetext{
* Valores sugeridos pela Nutron Alimentos Ltda., em trabalho colaborativo com o Grupo de Pesquisa em Conforto Ambiental, da Faculdade de Engenharia Agrícola - UNICAMP.
} 
box, sendo as imagens continuamente gravadas em um computador, por meio de placa de captura de vídeo que conectava as câmeras ao computador.

As matrizes pesadas foram monitoradas pelas câmeras de vídeos durante todo o período de luz, porém, para as observações dos comportamentos, foram utilizadas amostras de vídeos de 15 min pela manhã (6h01-12h), antes do arraçoamento, e de 15min pela tarde (12h01-18h), depois do arraçoamento, adaptando-se a metodologia descrita por BIZERAY et al. (2002). O pesquisador assistiu aos vídeos e as ocorrências dos comportamentos observados foram anotadas.

Comportamentos observados: a observação de todos os comportamentos mostrados na Tabela 1 deu-se por meio dos vídeos gravados a partir das câmeras de vídeo instaladas sobre os boxes.

TABELA 1. Etograma dos comportamentos observados.

\begin{tabular}{ll}
\hline Comportamento & Descrição \\
\hline Abrir asas & Movimento em que a matriz bate as duas asas \\
\hline Arrepiar penas & Ação de arrepiar e sacudir todas as penas do corpo \\
\hline Ciscar & $\begin{array}{l}\text { Movimento de arrastar a cama para trás com as patas e explorar a cama com o } \\
\text { bico. }\end{array}$ \\
\hline Correr & $\begin{array}{l}\text { Movimentação de uma matriz entre dois pontos distantes em velocidade maior } \\
\text { do que a observada normalmente. }\end{array}$ \\
\hline Deitar & Ato de a matriz ficar sentada ou deitada sobre a cama. \\
\hline Espojar & Banho realizado pela matriz utilizando o substrato da cama. \\
\hline Espreguiçar & $\begin{array}{l}\text { Ato em que a matriz estica uma asa e uma perna, do mesmo hemisfério do } \\
\text { corpo. }\end{array}$ \\
\hline Limpar penas & $\begin{array}{l}\text { Ato em que a matriz arruma as penas com o bico, induzindo a liberação de } \\
\text { óleos nas glândulas encontradas na base das penas. }\end{array}$ \\
\hline
\end{tabular}

Delineamento estatístico: o trabalho descrito consiste em um experimento fatorial fracionado $3^{(3-1)}$ com medidas repetidas (avaliando-se os efeitos da temperatura, idade, ração e turno). As repetições, e não replicações, são assim chamadas devido ao fato de que as mesmas aves foram observadas durante três dias consecutivos, fornecendo, assim, seis observações repetidas ao longo do tempo: dia 1 - manhã; dia 1 - tarde; dia 2 - manhã; dia 2 - tarde; dia 3 - manhã, e dia 3 - tarde. $\mathrm{Na}$ Tabela 2, ilustra-se o delineamento.

TABELA 2. Layout do experimento proposto: fatorial fracionado com medidas repetidas.

\begin{tabular}{cccccccc}
\hline \multirow{2}{*}{ Idade } & \multirow{2}{*}{ Turno } & \multicolumn{2}{c}{$\mathrm{T} 1$} & \multicolumn{2}{c}{$\mathrm{T} 2$} & \multicolumn{2}{c}{$\mathrm{T} 3$} \\
\cline { 3 - 7 } & & $\mathrm{Dia}$ & $1 ; 2 ; 3$ & $\mathrm{Dia}$ & $4 ; 5 ; 6$ & $\mathrm{Dia}$ & $7 ; 8 ; 9$ \\
\hline \multirow{2}{*}{$\mathrm{Id} 1$} & $\mathrm{M}$ & $\mathrm{R} 1$ & $* * *$ & $\mathrm{R} 3$ & $* * *$ & $\mathrm{R} 2$ & $* * *$ \\
& $\mathrm{~V}$ & $\mathrm{R} 1$ & $* * *$ & $\mathrm{R} 3$ & $* * *$ & $\mathrm{R} 2$ & $* * *$ \\
\hline \multirow{2}{*}{$\mathrm{Id} 2$} & $\mathrm{M}$ & $\mathrm{R} 2$ & $* * *$ & $\mathrm{R} 1$ & $* * *$ & $\mathrm{R} 3$ & $* * *$ \\
& $\mathrm{~V}$ & $\mathrm{R} 2$ & $* * *$ & $\mathrm{R} 1$ & $* * *$ & $\mathrm{R} 3$ & $* * *$ \\
\hline \multirow{2}{*}{$\mathrm{Id} 3$} & $\mathrm{M}$ & $\mathrm{R} 3$ & $* * *$ & $\mathrm{R} 2$ & $* * *$ & $\mathrm{R} 1$ & $* * *$ \\
& $\mathrm{~V}$ & $\mathrm{R} 3$ & $* * *$ & $\mathrm{R} 2$ & $* * *$ & $\mathrm{R} 1$ & $* * *$ \\
\hline
\end{tabular}

Id1; Id2; Id3 - idades das aves; T1, T2, T3 - condições ambientais propostas; R1, R2, R3 - composições de ração; M, V - turno matutino e vespertino. Os asteriscos correspondem às freqüências de um determinado comportamento de um trio de matrizes observado durante 15 minutos.

Análise e modelagem dos dados: com todas as variáveis estruturadas sob o delineamento proposto, fez-se, então, a análise confirmatória, utilizando os programas computacionais Minitab ${ }^{\circledR}$ e $\mathrm{SAS}^{\circledR}$. 
Foi utilizada a metodologia de modelos de Equações de Estimação Generalizadas (EEG), que incorpora a estimativa dos dados provenientes da mesma unidade experimental ao longo do tempo (medidas repetidas). Esse tipo de modelagem não exige que haja independência entre os valores observados no experimento, tal como é pressuposto nos Modelos Lineares Generalizados. No entanto, na metodologia de EEG, pode-se estimar, por meio da Matriz de Correlação de Trabalho, a forma pela qual os dados repetidos se correlacionam ao longo do tempo (HARDIN \& HILBE, 2003).

Para este experimento, a Matriz de Correlação de Trabalho estimou as associações entre as repetições segundo o modelo Auto-Regressivo de primeira ordem, AR (1), também utilizado nos modelos de Séries Temporais. Isso implica que a estrutura de autocorrelação entre as repetições decai exponencialmente, porém a autocorrelação parcial apresenta um único valor, só existindo autocorrelação significativa entre as repetições adjacentes (BOX \& JENKINS, 1976).

A coleta das freqüências caracterizou os dados como sendo provenientes da distribuição de Poisson. Para modelar essas observações, foi necessário o uso da função de ligação logaritmo neperiano (Ln), tornando o modelo de regressão linear com relação aos parâmetros. Assim, modelou-se a média das frequiências dos comportamentos em função dos fatores controlados no experimento. As variáveis independentes (ou preditoras) foram: temperaturas $\left({ }^{\circ} \mathrm{C}\right)$, idades das aves (em semanas), tipos de ração (variável Dummy ${ }^{* *}$ ) e turnos de coleta (variável Dummy). A matriz de dados foi codificada e disposta sob a forma de Casela de Referência, permitindo a comparação entre os níveis das variáveis Dummy desse modelo. Nesse caso, a referência para a Dummy turno é o da tarde; já para a ração, a referência é a R3 (de $2.789 \mathrm{kCal} \mathrm{kg}^{-1}$ ).

As variáveis Dummy contribuíram nos modelos como fatores condicionantes, de forma que, ocorrendo determinados níveis de um fator, essas variáveis contribuem para acréscimo ou decréscimo de um valor médio na predição do Ln da frequiência dos comportamentos. As estimativas dos coeficientes foram obtidas por meio do método iterativo modificado de Newton Raphson (HARDIN \& HILBE, 2003).

Matricialmente, os modelos propostos podem ser escritos na forma: supondo que $Y_{i}$ são variáveis aleatórias com distribuição Poisson, com média $\lambda_{i}$, em que $i=1, \ldots, \mathrm{n}$, pode-se obter um modelo linear para os parâmetros, por meio da função de ligação Ln, sendo o modelo, descrito matricialmente, com parte sistemática, dado pela eq. (1):

$$
\operatorname{Ln}\left(\lambda_{\mathrm{i}}\right)=\mathrm{X}^{\mathrm{T}} \beta
$$

Note-se que $\lambda_{i}$ é uma matriz coluna $(54 \times 1)$, contendo a freqüência esperada de um determinado comportamento; $X^{T}$ é matriz $(54 \times 6)$ das variáveis regressoras, estruturada no modelo Casela de Referência, e $\beta$ é a matriz linha (6 x 1) dos parâmetros. Os seis parâmetros citados estão descritos no modelo teórico apresentado na eq.(2):

Ln (Freqüência esperada de comportamento $)=\beta_{0}+\beta_{1}$ Temperatura

$+\beta_{2}$ Idade $+\beta_{3(\text { Turno=Manhã) }}+\beta_{3\left(\text { Raẵão=R1:2810 } \mathrm{kCal} \mathrm{kg}^{-1}\right)}+\beta_{4\left(\text { Raç̃̃o=R2:2800 } \mathrm{kCal} \mathrm{kg}^{-1}\right)}$

$+\beta_{5}$ Temperatura $_{\text {(Turno=Manhã) }}+$ erro

Nesse modelo, são apresentados os parâmetros a serem estimados (beta): o primeiro parâmetro do modelo é o intercepto $\left(\beta_{0}\right)$, Ln da freqüëncia esperada de um determinado comportamento, para matrizes observadas no turno vespertino e alimentadas com ração R3, e os demais coeficientes relacionam-se com as variáveis independentes.

\footnotetext{
** As variáveis Dummy são discretas, assumindo somente valores zero (0) e um (1), sendo identificadas nas equações por meio dos índices subscritos entre parênteses, ou juntamente da variável temperatura, denotando, assim, a interação entre a variável Dummy e a temperatura.
} 
Quando se referem às variáveis Dummy (ração e turno), os coeficientes são os incrementos médios sobre o intercepto devido à determinada condição descrita pela própria variável Dummy. Por exemplo, no caso do período, o coeficiente dessa variável representa o acréscimo médio do Ln da frequiência média devido à coleta ter sido na manhã (pois a referência para essa variável é o período da tarde). Porém, para as variáveis regressoras numéricas (temperatura e idade), a interpretação dos coeficientes foi equivalente à interpretação de um modelo comum de regressão, pois foram introduzidas no modelo como sendo contínuas e não como classes ou níveis, correspondendo ao quanto se aumenta o Ln da freqüência comportamental, devido ao aumento de uma unidade, numa determinada variável regressora.

O coeficiente da interação tempo e turno denota a taxa de aumento do Ln da média de freqüência comportamental devido ao aumento de uma unidade na temperatura, condicionado às observações durante o turno matutino. Dessa forma, pode-se avaliar se existe diferença no coeficiente angular da temperatura entre o turno da manhã e o da tarde que, quando ocorre, é dito que o efeito que a temperatura provoca no comportamento, depende do período em que se observaram as aves.

Ressalta-se que o efeito do turno é reflexo da escassez do alimento por tempo prolongado (quase 24 horas) - turno da manhã - ou o reflexo do pós-arraçoamento, havendo saciedade das aves durante a observação comportamental.

Todos os modelos dos respectivos comportamentos foram submetidos aos testes dos parâmetros, averiguando, assim, a significância do efeito que esses fatores produziram no comportamento, em nível de significância de 0,05.

O quociente Deviance/Gl correspondeu à estatística que mede a adequação do modelo ajustado (quanto mais próximo de um, mais bem ajustado é o modelo). Observa-se que todos os modelos possuem esse valor abaixo de 2, denotando a eficácia do modelo para os dados coletados (HARDIN \& HILBE, 2003). O Deviance mediu o desvio (ou distância) entre os valores ajustados pelo modelo e dos valores observados, semelhante ao teste Qui-quadrado de adequação do ajuste (HARDIN \& HILBE, 2003). O Gl (Grau de liberdade do modelo) foi calculado com o número de observações totais (54) subtraído pelo número de parâmetros estimados.

As equações selecionadas para os cenários foram referentes aos comportamentos de "ciscar" e "arrepiar penas", por serem comportamentos comprovadamente influenciados pelas condições de bem-estar térmico e disputa por alimentos (GRAVES, 1982; ESTEVEZ et al., 2003; PEREIRA, 2005; PEREIRA et al., 2005; SALGADO, 2006). Os cenários foram obtidos sob a amplitude da coleta dos dados, de acordo com os modelos estimados e em função do experimento realizado.

\section{RESULTADOS E DISCUSSÃO}

Todos os comportamentos foram analisados aplicando a técnica descrita na metodologia. Dentre os comportamentos analisados, dois apresentaram modelos significativos para a sua predição e se destacaram:

- O "ciscar", por ser um comportamento que carrega características mais complexas e amplas do bem-estar, pois, dependendo do turno, o ato de "ciscar" torna-se potencial indicador de bemestar térmico e de agressividade (PEREIRA, 2005; PEREIRA et al., 2005; SALGADO, 2006).

- O "arrepiar penas", por ser indicador exclusivamente de bem-estar térmico, se correlacionado inversamente ao aumento de temperatura (BIZERAY et al., 2002; PEREIRA, 2005; PEREIRA et al., 2005; SALGADO, 2006).

Foram verificados os principais resultados de avaliação do modelo referente ao comportamento de "ciscar" (Tabela 3) e de "arrepiar penas" (Tabela 4). Nessas tabelas, são apresentadas as estimativas dos parâmetros, com seus respectivos erros-padrão e p-valores dos testes dos parâmetros. 
Todos os testes relativos aos parâmetros presentes mostraram-se altamente significativos devido à precisão e às pequenas magnitudes dos p-valores (para o nível de significância de 0,05 ). Os parâmetros que não estão presentes, não tiveram significância $(\alpha=0,05)$, isto é, não foram significativamente capazes de explicar a variabilidade de variável-resposta em questão.

TABELA 3. Avaliação do modelo para o comportamento de "ciscar".

\begin{tabular}{cccr}
\hline Parâmetro & Estimativa & Erro-Padrão & P-valor \\
\hline Intercepto & 3,852 & 0,585 & $<0,0001$ \\
Temp. & $-0,081$ & 0,018 & $<0,0001$ \\
Idade & $-0,026$ & 0,010 & 0,0140 \\
Turno & $-3,160$ & 0,714 & $<0,0001$ \\
Temp. x Turno & 0,102 & 0,028 & 0,0004 \\
\hline
\end{tabular}

"Temp." corresponde à temperatura; "Turno" é referente ao da manhã (dummy); "Temp. x Turno" corresponde à interação da temperatura condicionada ao turno da manhã.

TABELA 4. Avaliação do modelo para o comportamento de "ciscar".

\begin{tabular}{cccc}
\hline Parâmetro & Estimativa & Erro-Padrão & P-valor \\
\hline Intercepto & 1,910 & 0,245 & $<0,0001$ \\
Temp. & $-0,094$ & 0,016 & $<0,0001$ \\
\hline
\end{tabular}

"Temp." corresponde à temperatura.

Para melhor visualização, os modelos são expressos por meio das eqs.(3 e 4), como seguem:

Modelo de previsão da freqüiência média de ocorrência do comportamento de "ciscar"

$$
\begin{aligned}
& \operatorname{Ln}(\text { Ciscar })=3,8526-0,0811 \text { Temperatura }-0,0261 \text { Idade }-3,1604_{\text {(Turno=Manhã) }} \\
& +0,1020 \text { Temperatura } \\
& \text { Deviance } / \mathrm{Gl}=1,9350 \\
& \mathrm{Gl}=49 \\
& \alpha=5 \%
\end{aligned}
$$

\section{Modelo de previsão da freqüência média do comportamento de "arrepiar penas"}

Ln $($ Arrepiar Penas $)=1,9105-0,0948$ Temperatur a

Deviance $/ \mathrm{Gl}=1,2209$

$\mathrm{Gl}=52$

$\alpha=5 \%$

A eq.(4), referente ao comportamento de "arrepiar penas", sugere indicador de bem-estar exclusivamente térmico para as matrizes pesadas, pois, independentemente da presença ou ausência do alimento, não apresenta interação e não depende da faixa etária. Isso mostra que a influência da temperatura sobre esse comportamento não está condicionado a nenhum outro fator (daqueles apresentados no experimento).

Por outro lado, o comportamento "ciscar" [eq.(3)] deve ser analisado mais cautelosamente. Os turnos (manhã e tarde) apresentaram interação com a temperatura para esse comportamento, demonstrando que a freqüência de "ciscar" dependeu conjuntamente da temperatura e do turno em que a ave se apresenta, isto é, não se pode relacionar a incidência desse comportamento com a temperatura ambiente, sem levar em consideração se as aves já se alimentaram ou não, já que o turno representa, indiretamente, o período que antecede o arraçoamento (manhã) e o período posterior ao arraçoamento (tarde). 
As aves, antes de receberem a primeira (e única) ração do dia, ficavam ávidas por alimento, ciscando numa freqüência independente da temperatura. Porém, quando as aves já haviam se alimentado (turno da tarde), a frequiência de "ciscar" passa a ter forte associação negativa com a temperatura. De modo que há diminuição do ato de "ciscar" ao longo do aumento da temperatura, e essa relação está de acordo com vários autores que estudaram essa relação (MITCHELL, 1987; TEETER \& BELAY, 1996; PEREIRA, 2005; PEREIRA et al., 2005; SALGADO, 2006). Sucintamente, a eq.(3) permitiu a interpretação de que, durante período muito longo de ausência de ração, as aves dão prioridade maior à necessidade de saciar a fome e à necessidade de se termorregularem (TEETER \& BELAY, 1996; PEREIRA et al., 2005; SALGADO, 2006).

Pode-se dizer, também, segundo a eq.(3), que o comportamento "ciscar" sofre decréscimo ao longo do envelhecimento das matrizes, corroborando os relatos de ESTEVEZ et al. (2003). Ao encontro dos relatos de ESMAY (1982) e de PEREIRA et al. (2005), as aves mais velhas e, portanto, maiores e mais pesadas, tendem a "ciscar" menos, fato observado pelo coeficiente negativo dessa variável.

Por meio da frequiência de "ciscar", pôde-se obter bom indicador térmico, desde que não haja escassez de alimento (turno da manhã). Durante a tarde, turno pós-arraçoamento, com as aves saciadas, a expressão do comportamento de "ciscar" passa a ter forte correlação inversa ao aumento de temperatura. Contudo, no período da manhã, a freqüência de "ciscar" não se associou significativamente à temperatura. Por esse motivo, somente no turno da tarde, a frequiência de "ciscar" deve ser utilizada como indicador mais sensível à temperatura ambiente, quando o arraçoamento se der no turno da manhã. Entretanto, no período de pré-arraçoameno (turno da manhã), quando há escassez de alimento, a freqüência de "ciscar" é inversamente correlacionada aos comportamentos agressivos entre as aves. Ou seja, com o aumento da frequiência desse ato, durante a falta de alimento, existe a diminuição de comportamentos agressivos, concordando com diversos autores (MARCHANT et al., 2001; PEREIRA, 2005; PEREIRA et al., 2005; SALGADO, 2006).

Assim sendo, considerou-se o indicador térmico mais adequado, aquele cujo modelo não obteve interação significativa, que foi o "arrepiar penas". Nota-se que a modelagem para tal comportamento não dependeu de outras variáveis, senão da temperatura ambiente, conforme ilustra a eq.(4).

Na Tabela 5, podem-se constatar os resultados dos diferentes cenários, utilizando a eq.(3). As mudanças de cores correspondem às mudanças de conforto térmico geral, devido à temperatura: cor cinza-claro - pouco estresse pelo frio; cor branca - zona de conforto térmico; cor cinza-escuro estresse térmico.

Segundo CURTIS (1983), ALBRIGHT (1990) e OLIVEIRA et al. (2005), a temperatura de maior conforto térmico, para aves em fase de postura, é $22,8{ }^{\circ} \mathrm{C}$. Baseando-se nesse resultado, a freqüência do comportamento de "ciscar" esperada, nos turnos matutino e vespertino, pode ser observada na Tabela 6, discriminando-se turnos e idades, configurado para o grupo de três matrizes, observadas durante $15 \mathrm{~min}$.

$\mathrm{Na}$ Tabela 7, podem-se verificar os resultados dos diversos cenários, utilizando-se da eq.(4), que depende apenas da temperatura. Tomando como base a mesma temperatura $\left(22,8{ }^{\circ} \mathrm{C}\right)$, a freqüência média de "arrepiar penas" ficaria em $0,78 \mathrm{vez}$, para um grupo do experimento, independentemente da idade e do turno.

Para o intervalo de zona de conforto térmico (de 20 a $26{ }^{\circ} \mathrm{C}$ ) aplicado na Tabela 7 , o número esperado de "arrepiar penas", adequado à condição de conforto térmico, é de 0,57 a 1,01 vez em média (durante 15mim). Assim, considerando as condições experimentais expostas na Tabela 7, quanto mais afastada estiver a freqüência média de "ciscar" (acima ou abaixo desse intervalo), maior será o gasto energético para essas aves se termorregularem, afetando negativamente a conversão alimentar e, conseqüentemente, a produtividade (MITCHELL, 1987; TEETER \& BELAY, 1996). 
TABELA 5. Resultados do cenário do comportamento de "ciscar".

\begin{tabular}{cccc}
\hline Temperatura $\left({ }^{\circ} \mathrm{C}\right)$ & Idade $($ semanas $)$ & Turno & Freqüência Esperada de "ciscar" \\
\hline 12 & 38 & manhã & 0,95 \\
13 & 44 & manhã & 0,83 \\
14 & 56 & manhã & 0,62 \\
12 & 38 & tarde & 6,60 \\
13 & 44 & tarde & 5,21 \\
14 & 56 & tarde & 3,51 \\
20 & 38 & manhã & 1,13 \\
23 & 44 & manhã & 1,02 \\
26 & 56 & manhã & 0,80 \\
20 & 38 & tarde & 3,45 \\
23 & 44 & tarde & 2,31 \\
26 & 56 & tarde & 1,33 \\
31 & 38 & manhã & 1,42 \\
33 & 44 & manhã & 1,26 \\
35 & 56 & manhã & 0,96 \\
31 & 38 & tarde & 1,41 \\
33 & 44 & tarde & 1,03 \\
35 & 56 & tarde & 0,64 \\
\hline
\end{tabular}

TABELA 6. Freqüência esperada de ciscar em temperatura ótima para o bem-estar térmico.

\begin{tabular}{ccc}
\hline & \multicolumn{2}{c}{ Temperatura $=2{ }^{\circ} \mathrm{C} \mathrm{a} 26{ }^{\circ} \mathrm{C}$} \\
Idade (semanas) & Turno & Freqüência Esperada de "ciscar" \\
\hline 38 & & 1,19 \\
44 & manhã & 1,02 \\
56 & & 0,75 \\
\hline 38 & & 2,75 \\
44 & tarde & 2,35 \\
56 & & 1,72 \\
\hline
\end{tabular}

TABELA 7. Resultados de simulação do comportamento de "arrepiar penas".

\begin{tabular}{cc}
\hline Temperatura $\left({ }^{\circ} \mathrm{C}\right)$ & Freqüência Esperada de "arrepiar penas" \\
\hline 12 & 2,17 \\
14 & 1,79 \\
16 & 1,48 \\
18 & 1,23 \\
20 & 1,01 \\
22 & 0,84 \\
24 & 0,69 \\
26 & 0,57 \\
28 & 0,48 \\
30 & 0,39 \\
32 & 0,33 \\
34 & 0,27 \\
36 & 0,22 \\
\hline
\end{tabular}


De acordo com ESMAY (1982), CURTIS (1983), ALBRIGHT (1990) e TEETER \& BELAY (1996), a exposição a baixas temperaturas tende a aumentar a produção de calor sensível, o que é resultado do acréscimo de metabolismo. Isso justifica a relação inversa entre a frequiência de "ciscar" e a temperatura ambiente, e também a relação inversa com a idade, uma vez que as aves mais jovens têm metabolismo mais alto que as mais velhas; e, ainda, quanto mais intenso for o metabolismo, maior o consumo e a necessidade de "ciscar", procurando reduzir a área de exposição à perda de calor sensível.

A freqüência de "arrepiar penas" está inversamente relacionada à temperatura ambiente, uma vez que esse comportamento é utilizado como mecanismo de isolamento térmico, criando uma camada de ar entre a superfície das penas e a pele da aves, evitando perda de calor sensível.

\section{CONCLUSÕES}

O monitoramento remoto de matrizes pesadas permitiu estudar o bem-estar térmico, com base em variações comportamentais específicas. Por meio das equações de predição e suas simulações, obteve-se panorama dos indicadores de bem-estar frente à temperatura ambiente, por meio das freqüências dos comportamentos de "ciscar" e "arrepiar penas".

\section{REFERENCIAS}

ALBRIGHT, L.D. Environmental control for animals and plants. St. Joseph: ASAE, 1990. 276 p.

BIZERAY, D.; ESTEVEZ I.; LETERRIERA, C.; FAURE, J.M. Effects of increasing environmental complexity on the physical activity of broiler chickens. Applied Animal Behaviour Science, Londres, v.79, n.1, p.27-41, 2002.

BOX, G.E.P.; JENKINS, G.M. Time series analysis: forecasting and control. San Francisco: Holden-Day, 1976. 375 p.

BOUSHY, A.R.; RATERINK, R. Eggshell strength: the cause of egg breakage in relation to nutrition, management and environment. Feedstuffs, Minneapolis, v.57, n.34, p.7-9, 1985.

BROWN-BRANDL, T.M.; JONES, D.D.; WOLDT, W.E. Evaluating modeling techniques for cattle heat stress prediction. Biossystems Engineering, Silsoe, v.91, n.4, p.513-24, 2005.

CAMPOS, E.J. O comportamento das aves. Revista Brasileira de Ciência Avícola, Campinas, v.2, n.2, p.93-113, 2000.

CURTIS, S.E. Environmental management in animal agriculture. Ames: The Iowa State University Press, 1983. 409 p.

CHENG, H.; MUIR, W.M. The effects of genetic selection for survivability and productivity on chicken physiological homeostasis. World's Poultry Science Journal, Bristol, v.61, n.3, p.383-98, 2005.

CURTO, F.P.F.; NÄÄS, I.A.; PEREIRA, D.F.; SALGADO, D.D. Estimativa do padrão de preferência térmica de matrizes pesadas (frango de corte). Revista Brasileira de Engenharia Agrícola e Ambiental, Campina Grande, v.11, n.2, p.211-16, 2007.

DUNCAN, I.J.H.; MENCH, J.A. Behaviour as an indicator of welfare in various systems. In: EUROPEAN SYMPOSIUM ON POULTRY WELFARE, 4., 1993, Potter Bar. Proceedings ... Potters Bar: Universities Federation for Animal Welfare, 1993. p.68-80.

ESMAY, M.L. Principles of animal environment.: Westport: AVI, 1982. 325 p.

ESTEVEZ I.; KEELING L.J.; NEWBERRY, R.C. Decreasing aggression with increasing group size in young domestic fowl. Applied Animal Behaviour Science, Londres, v.84, n.3, p.213-18, 2003. 
GRAVES, H.B. Behavioral responses of poultry (chickens) to management systems. In: SYMPOSIUM OF MANAGEMENT OF FOOD PRODUCING ANIMALS, 1982, West Lafayette. Proceedings... West Lafayette: Purdue University, 1982. v.2, p.122-38.

HARDIN, J.W.; HILBE, J.M. Generalized estimating equations. Boca Raton: Chapman \& Hall/CRC, 2003. 238 p.

KEER-KEER, S.; HUGHES, B.O.; HOCKING, P.M.; JONES, R.B. Behavioural comparison of layer and broiler fowl: measuring fear responses. Applied Animal Behaviour Science, Londres, v.49, n.4, p.321-33, 1996.

MARCHANT, J.A.; ANDERSEN, H.J.; ONYANGO, C.M. Evaluation of an imaging sensor for detecting vegetation using different waveband combinations. Computers and Eletronics in Agriculture, Bristol, v.32, n.1, p.101-17, 2001.

MARÍA, G.A.; ESCÓS, J.; ALADOS, C.L. Complexity of behavioural sequences and their relation to stress conditions in chickens (Gallus gallus domesticus): a non-invasive technique to evaluate animal welfare. Applied Animal Behaviour Science, Londres, v.86, n.1, p.93-104, 2004.

McGARY, S.; ESTEVEZ, I.; RUSSEK-COHEN, E. Reproductive and agressive behaviour in male broiler breeders with varying fertility levels. Applied Animal Behaviour Science, Londres, v.82, n.1, p.29-44, 2003.

MITCHELL, M.A. Some physiological effects of environmental temperature upon poultry. In: INTERNATIONAL POULTRY BUILDINGS CONFERENCE, 3., 1987, Londres. Proceedings... Londres, 1987. p.24-5.

NÄÄS, I.A. Aspectos físicos da construção no controle térmico do ambiente das instalações. In: CONFERÊNCIA APINCO, CIÊNCIA E TECNOLOGIA AVÍCOLA, 1994, Campinas. Anais... Campinas: FACTA, 1994. p.167.

OLIVEIRA, H.L.; AMENDOLA, M.; NÄÄS, I.A. Estimativa das condições de conforto térmico para avicultura de postura usando a teoria dos conjuntos Fuzzy. Engenharia Agrícola, Jaboticabal, v.25, n.2, p.300-7, 2005.

PEREIRA, D.F. Avaliação do comportamento individual de matrizes pesadas (frango de corte) em função do ambiente e identificação da temperatura crítica máxima. 2003. 174 f. Dissertação (Mestrado em Construções Rurais e Ambiência) - Faculdade de Engenharia Agrícola, Universidade Estadual de Campinas, Campinas, 2003.

PEREIRA, D.F. Metodologia para estimativa de bem-estar de matrizes de frango de corte utilizando monitoramento digital e construção de modelos de simulação. $2005.138 \mathrm{f}$. Tese (Doutorado em Construções Rurais e Ambiência) - Faculdade de Engenharia Agrícola, Universidade Estadual de Campinas, 2005.

PEREIRA, D.F; NÄÄS, I.A.; ROMANINI, C.E.B.; SALGADO, D.D.; PEREIRA, G.O.T. Indicadores de bem-estar baseados em reações comportamentais de matrizes pesadas. Engenharia Agrícola, Jaboticabal, v.25, n.2, p.308-14, 2005.

SALGADO, D.D. Modelo estatístico para predição de bem-estar de reprodutoras de frango de corte baseado em dados de ambiente e análise do comportamento. 2006. $113 \mathrm{f}$. Dissertação (Mestrado em Construções Rurais e Ambiência) - Faculdade de Engenharia Agrícola, Universidade Estadual de Campinas, 2006.

TEETER, R.G.; BELAY, T. Broiler management during acute heat stress. Animal Feed Science Technology, Londres, v.58, n.1, p.127-42, 1996.

TINÔCO, I.F.F. Ambiência e instalações na produção de matrizes avícolas. In: SILVA, I.J.O. Ambiência na produção de aves em clima tropical. Jaboticabal: SBEA, 2001. v.2, p.1-74. 